\title{
Epilepsy Focus Localization in Patients Utilizing BOLD Differences Related to Regional Metabolic Dynamics
}

\author{
Byron Bernal1,2,3*, Magno R. Guillen 1,2, Pedro Valdes³, Prasanna Jayakar'1, Nolan Altman², \\ Michael Duchowny ${ }^{1}$, Jorge Riera ${ }^{4}$
}

${ }^{1}$ Brain Institute, Nicklaus Children's Hospital, Miami, FL, USA

${ }^{2}$ Radiology Department, Miami, FL, USA

${ }^{3}$ Department of Pediatric, School of Medicine, Florida International University, Miami, FL, USA

${ }^{4}$ Department of Biomedical, School of Medicine, Florida International University, Miami, FL, USA

Email: *Byron.bernal@gmail.com

How to cite this paper: Bernal, B., Guillen, M.R., Valdes, P., Jayakar, P., Altman, N., Duchowny, M. and Riera, J. (2019) Epilepsy Focus Localization in Patients Utilizing BOLD Differences Related to Regional Metabolic Dynamics. Open Journal of Radiology, 9, 163-175.

https://doi.org/10.4236/ojrad.2019.93015

Received: July 18, 2019

Accepted: August 26, 2019

Published: August 29, 2019

Copyright $\odot 2019$ by author(s) and Scientific Research Publishing Inc. This work is licensed under the Creative Commons Attribution International License (CC BY 4.0).

http://creativecommons.org/licenses/by/4.0/

\begin{abstract}
This paper presents a theoretical elaboration aimed to explain the correlation found between a new rs-fMRI modality and electrophysiology and nuclear medicine neuroimaging, performed to localize epileptogenic brain areas. We present in detail the clinical history, and electrophysiological and neuroimaging results of one child with intractable epilepsy, who was submitted for Phase- 1 work-up as candidate for epilepsy surgery. The patient underwent a thorough workup including video-telemetry, ictal and interictal nuclear medicine imaging, resting-state fMRI, EEG-fMRI, intracranial electroencephalography (ECoG), deep electrode implantation, and resective surgery. Electrophysiology and neuroimaging findings were concordant with findings provided by the resting-state mean signal. The patient became seizure-free after the resection of the target area. A theoretical discussion is provided that considers the presence of a stable BOLD effect explaining the findings of the observed resting-state mean signal. This stable BOLD is linked to low regional metabolism usually present in the epileptogenic area during interictal periods, coupled with low oxygen extraction. Low oxygen extraction leaves more oxygen for the draining venule and consequently increases the BOLD signal.
\end{abstract}

\section{Keywords}

BOLD, fMRI, rs-fMRI, Brain, MRI, Epilepsy, PET

\section{Background}

The blood-oxygenation-level-dependent (BOLD) effect was initially described by 
Ogawa and co-workers in 1992 [1]. This physiological response of the brain tissue to external and endogenous stimuli has been the foundation for the MRI-based functional neuroimaging, which has led to the most fruitful period in modern neuroscience. Before the discovery of the BOLD effect, functional neuroimaging was limited to positron emission tomography (PET) and single photon emission computed tomography (SPECT), two techniques requiring radioactive tracers and characterized by poor spatial resolution. The discovery and posterior development of BOLD-sensitive sequences in MRI signified the advent of functional MRI, functional connectivity, regional homogeneity, and more recently, the discovery of the spontaneous brain fluctuations and the resting state networks. All of these functional assessment techniques are non-invasive as they do not require administration of contrast or radioactive agent.

The BOLD signal is generated due to local magnetic changes occurring in the oxyhemoglobin/deoxyhemoglobin ratio. Deoxyhemoglobin is a paramagnetic molecule, and its presence increases the de-phasing of the transversal magnetization by a local field inhomogeneity interaction reducing the $\mathrm{T}_{2}$ signal. Therefore, those areas with less deoxyhemoglobin (that is, with the highest tissue oxygen pressure) will have longer-lasting $\mathrm{T}_{2}$ signal. From this description, two main factors suffice to explain the deoxyhemoglobin changes at the venule level responsible for the BOLD signal: oxygen supply and oxygen extraction (See series of Equations). Oxygen supply is a function of hemoglobin availability (by far the main carrier) and cerebral blood flow (CBF); oxygen extraction depends mostly on the metabolic rate of the neuron. Since the hemoglobin level of a given subject depends on the hematocrit which should be assumed constant between distinct brain regions, and within the same session, we may assume that the BOLD effect rely on the interrelation between two main factors: CBF and regional metabolism. These two factors may be combined into a concept that explains the signal variation in BOLD sensitive series.

\section{The Concept of Vascular/Metabolic Ratio}

It is currently established that most of the variability of the BOLD effect is explained by the vascular factor; stated as follows: A stimulated brain area increases adenosine triphosphate (ATP) consumption. In neurons, ATP is mainly produced in the mitochondria through a phosphorylation process requiring oxygen [2]. As a consequence, cerebral metabolic rates of oxygen $\left(\mathrm{CMRO}_{2}\right)$ increases and partial oxygen pressure in brain tissue drops. Simultaneously, a diversity of neural and glial mechanisms trigger locally the release of vasoactive substances in the activated brain area [3] [4]; resulting in an increase in regional cerebral blood flow $(\mathrm{CBF})$. However, vascular and metabolic increases are not proportional. CBF increases 2 to 4 times more than $\mathrm{CMRO}_{2}$ ratio [5]. Increases in this ratio above the level of the background average yields the source of the functional MRI activation signal. The following equation characterizes the MRI $\mathrm{T}_{2}{ }^{*}$ weighted signal strength causing the BOLD. 


$$
S o=M o * \mathrm{e}^{\left(-T_{E} R_{2}\right)}
$$

where, So is the signal strength, Mo is the initial magnetic field strength, $e$ is the base of the natural logarithm (Euler's number), $T_{E}$ is the echo time, and $R_{2}$ is a dynamic parameter that reflects relaxation properties affected by local magnetic field inhomogeneities (that is, proportional to the concentration of deoxyhemoglobin). It is worth noting that the strength of the MRI signal $S$ is inversely correlated with $R_{2}$ in an exponential manner, that is, the lower $R_{2}$ the higher MRI signal $S$.

In this paper, we will describe a case with neuroimaging findings suggesting a BOLD effect depending mostly upon a regional pathological sustained metabolic change, and how it eventually affects the venular oxygen, and consequently the MRI signal strength. Our aim is not to prove our hypothesis but to provide solid basis to elicit further research on the study of this stable BOLD effect allegedly related to a brain pathology.

\section{Exemplary Case}

\subsection{The Observation}

Patients with drug-resistant (intractable) epilepsy should be assessed for candidacy of surgical treatment. The usual work-up in those cases includes eloquent brain areas mapping. As expected, many of these candidates are children, as it has been proven that surgical interventions for intractable epilepsy are of better prognosis when performed at early ages [6]. However, children younger than 8 years of age show limited cooperation.

The lack of cooperation of small children and patients with cognitive deficits to cope with task-related fMRI poses a pragmatic problem in the attempt to map their critical functions. For these reasons, the Independent Component Analysis (ICA) of the rs-fMRI has been utilized in different clinical centers to localize sensory-motor [7], visual [8], and language functions [9] [10].

One intermediate file obtained during the rs-fMRI processing with FSL MELODIC is the "mean" file; plainly, a signal average across the time points of the rs-fMRI sequence. An incidental finding observed in these images was the lack of homogeneity between different cortical areas and the asymmetry between homotopic areas when the image was given a color look-up table (Figure 1).

Further observations of the same type encouraged our team to conduct a more systematic observation. In 2015, we published the results of a study comparing two groups: 29 epilepsy patients and 22 control subjects [11]. Inter-group comparisons showed that the mean intensity between the "means"-normalized to the invariant intensity of the CSF-differed significantly $(\mathrm{p}<0.001)$. Patient group showed also significant greater intra volume variance $(\mathrm{p}<0.001)$ as compared to normal subjects. In a further analysis, we compared the Euclidean distance (ED) between the isocenter of the "mean" hyperintensity to the isocenter of the suspicious epileptogenic area as demonstrated by a Standard Reference, that for the sake of that study was either an Ictal SPECT or an interictal PET. An overlap was 


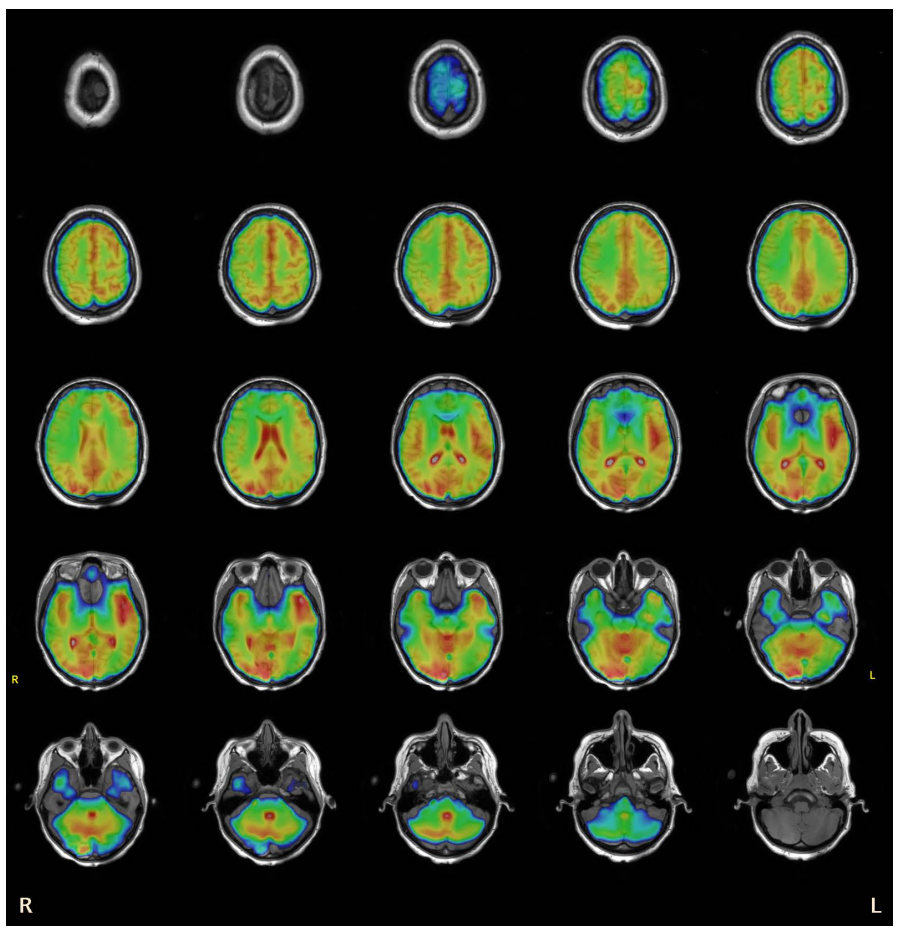

Figure 1. Resting-state "Mean" series. Intensity signal from the source image has been color coded utilizing a "spectrum" lookup table. Comparison of interhemispheric homotopic brain areas reveal overt signal asymmetries.

accepted as a match if the ED was less than $5 \mathrm{~cm} .72 .4 \%$ of them had matching overlap.

In the same report, we speculated about the origin of this finding suggesting 3 hypotheses:

1) The effect of a localized neural oscillation with higher point of inflection.

2) A transient BOLD effect related to the inter-ictal epileptic discharges.

3) A plain $T_{2}$ local effect.

The hypotheses were discussed in the paper, and were rejected based on the following facts.

The effect of a localized neural oscillation would have a representation in a "neural component" on the ICA with the same localization. These matching networks only appeared in a very small number of cases. Moreover, a localized interictal higher base-line oscillation would necessarily be coupled with hypermetabolism in PET studies. However, the vast majority of our cases (allegedly performed within the inter-ictal phase) showed also hypo-metabolism in the interictal PET study as it was expected to occur in the epileptogenic zone. The effect of a transient BOLD related to the interictal epileptic discharges was also ruled out because these transients are of small duration and signal variation is tiny. To extract the BOLD effect in these cases, it is necessary to convolve the data with time-locked regressors derived from the interictal epileptic events. In order for the interictal discharges to become hyper intense per se, they require to be quite frequent, in which case the PET should become hypermetabolic. 
The hypothesis that the "mean" is due to a mere $\mathrm{T}_{2}$ effect that may be related to high intensity of focal cortical dysplasia, cysts, CSF and parenchymal edema seemed tempting. However, the high intensity signal of the "mean" was clear even in non-lesional cases, in which by definition the $T_{2}$ sequences are normal. In addition, several observations have shown a lack of relationship between the multiple $\mathrm{T}_{2}$-FLAIR hyperintensities seen in cases of tuberous sclerosis and the hyperintensities shown by the "mean". Still, one can argue that there are minute tissular changes not detectable when the image is plain in grey scale and that they become overt thanks to the color code spread given by the look-up table applied to the image. Isolated exemplary cases have shown us this is not the case. Figure 2 shows a $\mathrm{T}_{2}$-FLAIR image of another patient depicting areas of hyperintensity, related to tuberous sclerosis, which are not all concordant with those shown in the "mean". In fact, the left frontal tuber that exhibits a lower intensity peak than the right parietal in the $\mathrm{T}_{2}$-FLAIR image shows a reverse pattern in the "mean" image. Moreover, we have found cases in which the "mean" changes between sessions reflecting functional dynamics that cannot be explained by structural changes (Figure 3).
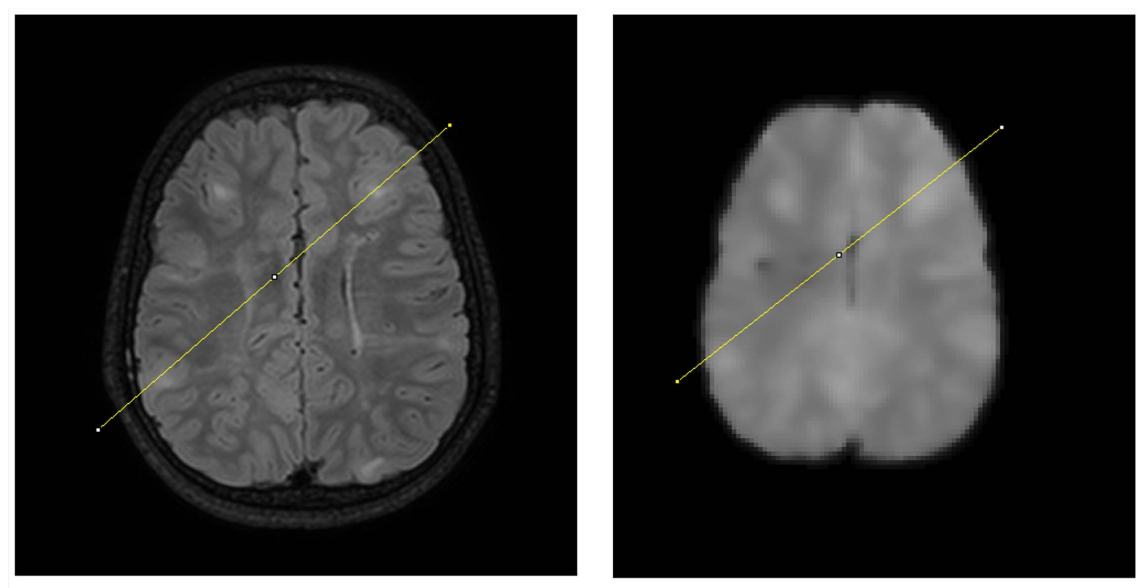

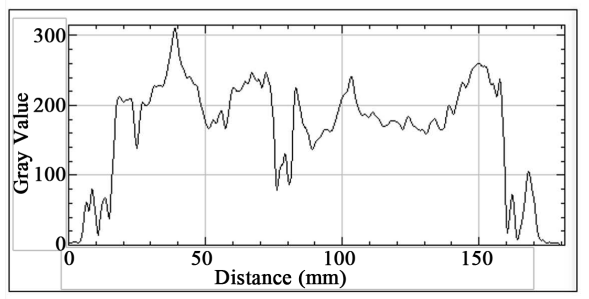

(a)

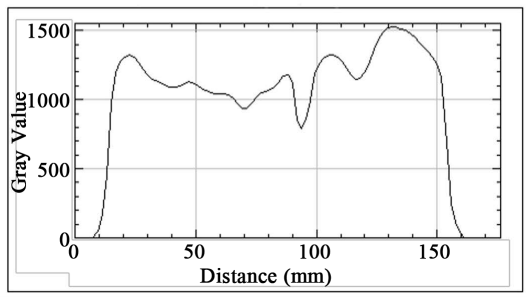

(b)

Figure 2. FLAIR vs "Mean" comparison of intensities in an exemplary case with Tuberous Sclerosis. Two radiological-oriented axial corregistered planes are shown: $\mathrm{T}_{2}$-FLAIR (a), and "mean" (b). A line crossing the hemispheres intersecting two tubers have been placed symmetrically in each hemisphere. In the lower row, the depiction of the intensities belonging to voxels in the line is shown. The comparison shows similar but not identical profiles. In the FLAIR image the maxima value is located in the right hemisphere (left half of the curve); in the "mean" the maxima value is located in the left hemisphere (right half of the curve). Peaks and valleys within the profiles are non-concordant. This strongly suggests that "mean" intensities do not represent a mere $\mathrm{T}_{2}$ effect. 

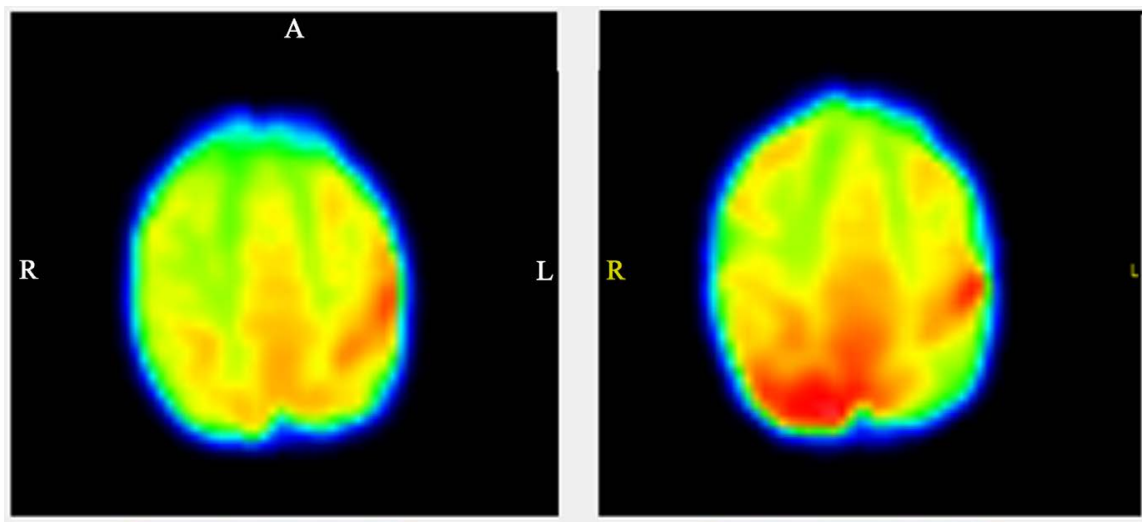

Figure 3. Comparison of "Mean" axial cuts of the same patient acquired in two different times the same day. Note the changes of intensity observed in the right parietal region.

\section{A new hypothesis}

Most recently, we have arrived to a new hypothesis that harmonizes theory and empirical observations between neuroimaging and neurophysiology studies. In the following part, we will utilize the presentation of one exemplary case that will provide the logical path to elaborate our hypothesis on the foundations of the signal origin of the "mean" BOLD.

\subsection{Case Presentation}

Written informed consent was obtained from the patient's legal guardian for this publication. A 16-year-old right-handed girl, began having seizures at age 14 . Seizures consisted of behavioral arrest and upward rolling eyes. She also had some generalized tonic-clonic seizures without conscious aura. The first type of seizures is daily, frequent enough that "she had to go into the hospital homebound school program".

The patient's interictal wakeful EEG showed a background consisting of medium amplitude symmetric and synchronous $8-9 \mathrm{~Hz}$ rhythms with posterior dominance. There were "bilateral independent frontal and bi-frontal synchronous spike, polyspikes and slow wave discharges noted in awake and sleep". The ictal EEG showed "multiple abrupt onset of bi-frontal regular 3 - $4 \mathrm{~Hz}$ spikeand-slow-wave discharges with abrupt offset resembling a typical absence" (Figure 4, inset (d) "Interictal EEG").

EEG-source localization showed a right medial and central dipole in its earliest phase, then a rotating dipole localized in the right post-central areas.

The patient's MRI studies were performed in a Philips $1.5 \mathrm{~T}$ magnet, equipped with a 16 SENSE Rx channel coil. The standard MRI showed nonspecific haziness of the gray-white interface in the right middle frontal gyrus and right temporo-occipital region (Figure 4, inset (a) "Axial $\mathrm{T}_{1}$ and FLAIR").

The patient's fMRI showed left lateralization of language for both receptive and expressive areas in two distinct paradigms (Auditory description task and Antonyms/Synonyms discrimination task). Sensory/motor representation for hands and visual fMRI were normal. 


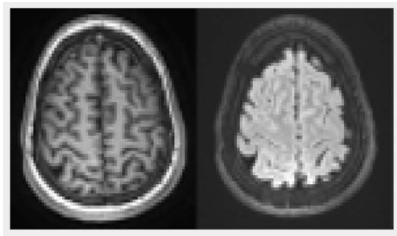

(a)

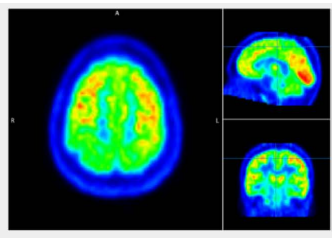

(b)

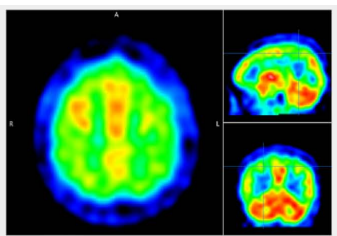

(c)

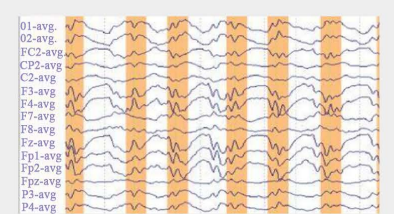

(d)

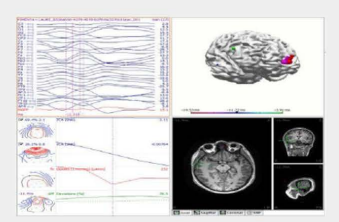

(e)

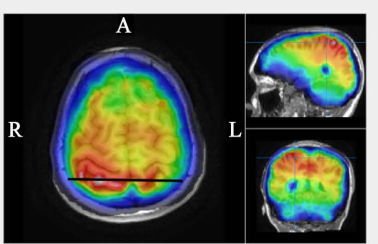

(f)

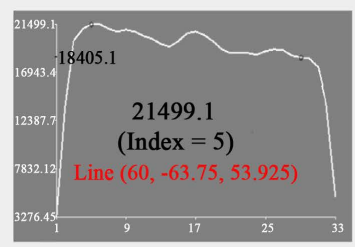

(g)

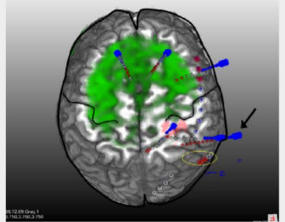

(h)

Figure 4. Neuroimaging work-up. (a) Axial T1 and FLAIR: Non lesional; (b) Interictal PET: right parietal hypometabolism; (c) Ictal SPECT: Right frontal hypermerabolism; (d) Interictal EEG: right > left frontal IED; (e) EEG source localization: right temp $\rightarrow$ frontal; (f) Mean-BOLD: Right parietal high BOLD signal; (g) Intensity profile of inline voxels in mean-bold; (h) Implantation: Positive findings in right parietal strip and depth electrode (arrow). Of note: All insets, except last one, show transversal cuts of the brain oriented in radiological convention (left hemisphere on the right side). The voxel intensities within the transversal line placed in inset ( $\mathrm{f}$ ) "Mean" are graphically represented in the curve shown in adjacent inset (g). Note the difference in signal intensity of two point equidistant from the sagittal midline. The number under the curve is the maximum value. Left side of the curve corresponds to right hemisphere. The last inset shows a parietal transversal cut in a $3 \mathrm{D}$ volume rendering of the brain. In this case, the right hemisphere appears where it should be.

The "mean" showed an area of well-defined local hyperintensity in the right parietal area, affecting both the convexital and paracentral aspects (Figure 4, inset (f) "Mean-Bold”).

The interictal PET showed a subtle "area of decreased metabolism in the right high central and post-central region" (Figure 4, inset (b) "Interictal PET"). The ictal SPECT showed increased uptake in the right frontal lobe, laterally. Increased uptake was also seen in the left cerebellar hemisphere (Figure 4, inset (c) "Ictal SPECT").

In addition to this information, the patient jointed a research protocol for simultaneous EEG-FMRI in the same Philips scanner. Four 10-minute trials sessions of $300 \mathrm{TR}=2 \mathrm{~s}$ timepoints were acquired utilizing the same protocol used for her prior rs-FMRI. In order to detect the interictal epileptic discharges (IED), EEG was recorded using a 10 - 10 system 32-channel EasyCap (Brain Amp MR, Brain Product $\mathrm{GmbH}$ ). In order to synchronize the EEG with the fMRI scans, a trigger signal marking the beginning of the scans was sent to the EEG recording 
laptop. This laptop was placed in the operator's room, and it was connected to the amplifiers via fiber-optics. The following EEG preprocessing was performed using BrainVision Analyzer 2 (Brain Products $\mathrm{GmbH}$ ). To remove the MR-related artifact, the EEG data was first subsampled to $50 \mathrm{kHz}$ using sync interpolation to virtually increase its resolution and correct the random phase jittering-of no less than $0.2 \mathrm{~ms}$ resolution determined by the $5 \mathrm{kHz}$ sampling rate-that is present in the scan markers. This phase jittering has significant negative effects in the estimation of the MR gradient artifacts since the latter can change as fast as $0.2 \mathrm{~ms}$. Subsequently, we applied a method for removing the MRI gradient artifacts, based on the estimation of an average artifact template. The resultant EEG data was bandpass filtered within $0.5-125 \mathrm{~Hz}$. After marking the $\mathrm{R}$-waves using a semiautomatic tool, we applied a method to detect and remove the effects of the balistocardiogram [12]. Finally, we applied ICA based on the infomax method [13] [14]; to remove further artifacts. Additionally, motion correction parameters were included as nuisance regressors. We then attempted to classify them into NBR mechanisms using the machine learning classifier. Finally, we estimated the parameters of the corresponding particular sub-model. (Figure 4, inset (e) "EEG source localization", see black arrow).

All the intra-session IED were identified and its time annotated. A regressor was built representing the time-location of the discharges. The data was processed utilizing a General Linear Model with SPM [15]. The voxels with significant correlation with the interictal epileptic discharges were color coded and overlaid on the Axial $T_{1}$ MRI. Main areas of correlations were found in the right precentral region.

All in all three possible IED sources were considered: Right parietal in keeping with the PET and EEG findings; the cingulate gyrus (either side) due to the long duration of absences and bifrontalictal onset; and right superior frontal gyrus in keeping with a suggestive signal increase in the SPECT.

With these results, the patient was implanted (Phase I) in the right parietal area. Two deep electrodes, 2 four-contact strips and one eight-contact strip were implanted targeting the right parietal convexity and both cingulate gyrus through burr holes. Stereo EEG and intraoperative electrocorticography was performed using a 64-channel digital system. Frequent monomorphic sharps were found in mid electrodes of one depth parietal electrode; there were also spike and slow wave in electrodes of the parieto-lateral strip and depth electrode. Several seizures episodes were recorded. Correlates of these seizures were buildup of spike and slow waves over the same electrodes followed by simultaneous involvement of neighbor electrodes (Figure 4, inset (h) "Implantation", see black arrow).

The patient underwent ECoG guided resective surgery with parietal and posterior cingulate gyrus resection. Since then (6 months and 15 days she has remained seizure free); an initial numbness experienced in the left hemibody has improved. There is no cognitive sequelae. 


\section{Discussion}

In a previous publication, we have showed that patients with epilepsy have resting-state "mean" images that differ from normal subjects [11]. In addition, local high regional intensities result concordant with the localization of PET or SPECT studies in $72 \%$ of epilepsy patients. The case presented here strongly suggests the relationship between interictal hypometabolism and an increase in the intensity of the area in the "mean" image provided by the rs-fMRI.

The reasons to rule out a BOLD effect given by the epileptic discharges acting as endogenous stimuli, and the effect of a localized spontaneous oscillation with higher point of inflection, have been explained above. The effect of $T_{2}$ tissular properties was also briefly analyzed but still is an important confound. The rs-fMRI mean will show as bright any structure with long $\mathrm{T}_{2}$. This obviously refers to water (protons), present in the CSF filling the ventricles and subarachnoideal spaces. All cystic cavities and areas with edema will also appear with high intensity. However, the sequences weighted with $\mathrm{T}_{2}{ }^{*}$ reflect earlier stages of the transversal magnetization due to local-field-inhomogeneity interactions. Throughout the entire brain, local field inhomogeneities may be explained as differences in neuron, axon, glia, vessel and interstitial fluid-changes which can occur qualitatively or quantitatively accordingly with the anatomy and the function of the area. However, we can assume that tissular variability is similar between homotopic areas, which will be expressed in neuroimaging as symmetrical findings. Much larger changes are expected from a moving tissue: blood. Venule blood shows changes depending upon two variables: blood flow and oxygen extraction.

In our exemplary case, the region with the highest resting state "mean" signal shows co-localized low interictal-PET signal (a negative correlation that was also found in the majority of our reported cases). As it was proved with the intracranial EEG, the area was the source of the interictal epileptic discharges. The negative correlation between the "mean" and PET has been confirmed in a case in whom a left frontal epileptic focus exhibited very frequent interictal discharges. As expected, the interictal PET showed hypermetabolism of the epileptic zone, while the "mean" showed an area of local low signal in the same region. Given the fact that the "mean" was obtained during resting state conditions, that is, in the absence of any stimulus for the patient, it seems logical to infer that the observation could be explained only as a consequence of regional metabolic changes. In summary, the hypothesis would be: Areas with low metabolism have increased "mean" (BOLD) signal.

To explain the underlying physiology of our hypothesis, we remit to equation 1, from which it can be observed that the strength of the MRI signal $S$ is inversely correlated with $R_{2}$ in an exponential manner; i.e., the lower $R_{2}$ the higher the $S$. Equation (2) explains the relationship between $R_{2}$ and deoxyhemoglobin. The sequence of equations that follows demonstrate the effect of the venular oxygen level and CBF in the MRI signal strength. 


$$
R_{2}=\mathrm{Area} * \mathrm{CBV} *[\mathrm{dHB}]
$$

where Area is the area of the blood vessel associated to the transversal section of the vessel (a measure of vasodilation), CBV is the cerebral blood volume, and $[\mathrm{dHB}]$ is the concentration of deoxyhemoglobin.

As a consequence of combining Equations (1) and (2), brain areas with more oxyhemoglobin (that is less deoxyhemoglobin) will have higher signal (and appear brighter) than those containing more deoxyhemoglobin as this molecule creates more magnetic inhomogeneity. Since the concentration of deoxyhemoglobin at the venule level $\left([\mathrm{dHB}]_{\text {ven }}\right)$ can be expressed in terms of net oxygen availability in the arteriole (oxygen supply), and oxygen extraction then follows:

$$
[\mathrm{dHB}]_{\mathrm{ven}}=\mathrm{OEF} * \mathrm{O}_{2}
$$

where $\mathrm{OEF}$ the oxygen extraction fraction and $\mathrm{O}_{2}$ represents the oxygen supply.

The OEF, CBF and oxygen supply are factors of the Cerebral Metabolic Rate of the Oxygen $\left(\mathrm{CMRO}_{2}\right)$ expressed in Equation (4):

$$
\mathrm{CMRO}_{2}=\mathrm{CBF} * A=\pi r^{2} * \mathrm{O}_{2}
$$

Replacing Equation (3) in Equation (4) we obtain:

$$
\mathrm{CMRO}_{2}=\mathrm{CBF} *[\mathrm{dHB}]_{\mathrm{ven}} \rightarrow[\mathrm{dHB}]_{\mathrm{ven}}=\frac{\mathrm{CMRO}_{2}}{\mathrm{CBF}}
$$

It can be observed that the concentration of deoxyhemoglobin at the venule level is directly proportional to the cerebral metabolic rate and inverse proportional to the CBF (Equation (5)).

Replacing factors of Equation (4) in (2):

$$
R_{2}=\mathrm{Area} * \mathrm{CBV} *\left(\frac{\mathrm{CMRO}_{2}}{\mathrm{CBF}}\right)
$$

Replacing factors of Equation (6) in Equation (1), it is clear the inverse correlation between signal strength of $\mathrm{T}_{2}{ }^{*}$ sequences ( $\mathrm{S}$ ) with $\mathrm{CMRO}_{2}$ (oxygen extraction) and the direct correlation to CBF.

Therefore, we think this non-stimulus-related BOLD effect is due to a functional change in blood oxygenation at the venule level which depends on the mismatch between the vascular $(\mathrm{CBF})$ and neuronal metabolism $\left(\mathrm{CMRO}_{2}\right)$ changes, assuming a constant hematocrit [16].

The chain of events we hypothesize is: low metabolic areas (epileptogenic zone during interictal periods) have reduced $\mathrm{CMRO}_{2}$, leaving more oxyhemoglobin for the venule, reducing deoxyhemoglobin ratio and therefore increasing the BOLD signal. Of importance is to note here that in contrast to the transient BOLD effect of the standard neuro-vascular coupling, the "mean" is a sustained change. In other words, the standard BOLD differs in time and region; the differences of the "mean" rely only on sustained regional changes.

The effect may be small but can be made overt with the help of spectrum look up tables that spread the intensity values into different hues. That is we think the "mean" is representing. In that sense our "mean" map is a "mean-BOLD", and 
the regional signal changes reflect not only the $T_{2}$ differences explained by the presence of water but, most importantly the regional changes due to variations of regional metabolism, that has been well described associated with epilepsy.

Metabolic regional changes are not exclusive of focal epilepsy. Any type of regional parenchymal pathology that affects the metabolism should also be reflected in the "mean-BOLD".

Certainly, the field of fMRI is vastly dominated by task-related fMRI. Notwithstanding, fMRI has evolved into a task-free type of functional brain mapping. rs-fMRI has demonstrated the presence of spontaneous low-frequency brain oscillations (of the $\mathrm{T}_{2}{ }^{*}$ signal) in the absence of external stimulus. The contribution of metabolic and vascular factors in the generation of the spontaneous brain oscillations is still a matter of research. Crucial questions are still pending to be resolved: are the spontaneous brain oscillations related to spontaneous regional vascular neuro coupling? Or, are they related to akin metabolic tides? The current view of this conundrum is that there is a direct proportionality between the resting-state BOLD signal and CBF responses. That means that neuro-vascular coupling maintains a spontaneous dynamic state under rest conditions.

More research is needed to understand completely the mean-BOLD effect. Some of our studies have shown that the hyperintensities are adjacent to areas where the epileptic discharges are demonstrated. We do not know the trajectories of the "mean-BOLD" in the immediate post-ical, the pre-ictal, and even the ictal periods. We do not know either if the effect is also present in adults. The outcome predictive value of the "mean-BOLD" is currently evaluated. Sixty per cent of subjects with brain resections that overlap the "mean-BOLD" hyperintense areas have remained seizure free for up to two years (report in preparation). This suggests that the "mean-BOLD" may reflect not only the epileptogenic areas but the symptomatogenic areas as well. Then it usefulness may be improved when its results are analyzed in combination with the EEG results and other modalities of epilepsy assessment.

\section{Conclusion}

Years of experience with rs-fMRI in children with intractable epilepsy have shown us that, in a significant group of patients, there is a correlation between localized changes in an image derived from this technique and the area that is eventually signaled as the epilepsy origin. The image is an average of the time-points of the rs-fMRI. We have called it the "mean-BOLD". In this paper, we present a physiological, mathematical and observational line of thought to propose that it is the result of a nonvascular dependent BOLD phenomenon that may be explained as a consequence of discrete and sustained regional changes of neuronal metabolism. Low metabolism, usually seen in the epileptogenic zone, is coupled with diminished oxygen extraction. As a direct result, more oxyhemoglobin is left in the draining venous side, explaining the regional Bold effect. It 
seems to us that because of its simplicity, lack of invasiveness, and availability, it may help clinicians to orient to localize epileptic origin of seizures.

\section{Acknowledgements}

This article is the product of a self-initiated, self-sponsored theoretical study.

\section{Conflicts of Interest}

The authors declare that this case report was conducted in the absence of any commercial or financial relationships that could be construed as a potential conflict of interest.

\section{References}

[1] Ogawa, S., Tank, D.W., Menon, R., Ellermann, J.M., Kim, S.G., Merkle, H., et al. (1992) Intrinsic Signal Changes Accompanying Sensory Stimulation: Functional Brain Mapping with Magnetic Resonance Imaging. Proceedings of the National Academy of Sciences of the United States of America, 89, 5951-5955. https://doi.org/10.1073/pnas.89.13.5951

[2] Erecińska, M. and Silver, I.A. (1989) ATP and Brain Function. Journal of Cerebral Blood Flow \& Metabolism, 9, 2-19. https://doi.org/10.1038/jcbfm.1989.2

[3] Logothetis, N.K. and Pfeuffer, J. (2004) On the Nature of the BOLD fMRI Contrast Mechanism. Magnetic Resonance Imaging, 22, 1517-1531.

https://doi.org/10.1016/j.mri.2004.10.018

[4] Wells, J.A., Christie, I.N., Hosford, P.S., Huckstepp, R.T.R., Angelova, P.R., Vihko, P., et al. (2015) A Critical Role for Purinergic Signalling in the Mechanisms Underlying Generation of BOLD fMRI Responses. Journal of Neuroscience, 35, 5284-5292. https://doi.org/10.1523/JNEUROSCI.3787-14.2015

[5] Buxton, R.B. (2010) Interpreting Oxygenation-Based Neuroimaging Signals: The Importance and the Challenge of Understanding Brain Oxygen Metabolism. Frontiers in Neuroenergetics, 2, 8. https://doi.org/10.3389/fnene.2010.00008

[6] Chen, H.-H., Chen, C., Hung, S.-C., Liang, S.-Y., Lin, S.-C., Hsu, T.-R., et al. (2014) Cognitive and Epilepsy Outcomes after Epilepsy Surgery Caused by Focal Cortical Dysplasia in Children: Early Intervention Maybe Better. Child s Nervous System, 30, 1885-1895. https://doi.org/10.1007/s00381-014-2463-y

[7] Roder, C., Charyasz-Leks, E., Breitkopf, M., Decker, K., Ernemann, U., Klose, U., et al. (2016) Resting-State Functional MRI in an Intraoperative MRI Setting: Proof of Feasibility and Correlation to Clinical Outcome of Patients. Journal of Neurosurgery, 125, 401-409. https://doi.org/10.3171/2015.7.JNS15617

[8] Vergun, S., Gaggl, W., Nair, V.A., Suhonen, J.I., Birn, R.M., Ahmed, A.S., et al. (2016) Classification and Extraction of Resting State Networks Using Healthy and Epilepsy fMRI Data. Frontiers in Neuroscience, 10, 440. https://doi.org/10.3389/fnins.2016.00440

[9] Tie, Y., Rigolo, L., Norton, I.H., Huang, R.Y., Wu, W., Orringer, D., et al. (2014) Defining Language Networks from Resting-State fMRI for Surgical Planning-A Feasibility Study. Human Brain Mapping, 35, 1018-1030. https://doi.org/10.1002/hbm.22231

[10] Lu, J., Zhang, H., Hameed, N.U.F., Zhang, J., Yuan, S., Qiu, T., et al. (2017) An Automated Method for Identifying an Independent Component Analysis-Based Lan- 
guage-Related Resting-State Network in Brain Tumor Subjects for Surgical Planning. Scientific Reports, 7, 13769. https://doi.org/10.1038/s41598-017-14248-5

[11] Bernal, B., Guillen, M.R., Zamora, K. and Altman, N. (2015) Utilizing rs-fMRI's Mean to Localize Seizure Focus. Open Journal of Radiology, 5, 92-103. https://doi.org/10.4236/ojrad.2015.52015

[12] Allen, P.J., Polizzi, G., Krakow, K., Fish, D.R. and Lemieux, L. (1998) Identification of EEG Events in the MR Scanner: The Problem of Pulse Artifact and a Method for Its Subtraction. NeuroImage, 8, 229-239. https://doi.org/10.1006/nimg.1998.0361

[13] Bell, A.J. and Sejnowski, T.J. (1995) An Information-Maximization Approach to Blind Separation and Blind Deconvolution. Neural Computation, 7, 1129-1159. https://doi.org/10.1162/neco.1995.7.6.1129

[14] Makeig, S., Jung, T.P., Bell, A.J., Ghahremani, D. and Sejnowski, T.J. (1997) Blind Separation of Auditory Event-Related Brain Responses into Independent Components. Proceedings of the National Academy of Sciences of the United States of America, 94, 10979-10984. https://doi.org/10.1073/pnas.94.20.10979

[15] Friston, K.J., Holmes, A.P., Worsley, K.J., Poline, J.-P., Frith, C.D. and Frackowiak, R.S.J. (1994) Statistical Parametric Maps in Functional Imaging: A General Linear Approach. Human Brain Mapping, 2, 189-210. https://doi.org/10.1002/hbm.460020402

[16] Davis, T.L., Kwong, K.K., Weisskoff, R.M. and Rosen, B.R. (1998) Calibrated Functional MRI: Mapping the Dynamics of Oxidative Metabolism. Proceedings of the National Academy of Sciences of the United States of America, 95, 1834-1839. https://doi.org/10.1073/pnas.95.4.1834 